\title{
Accuracy of the Actigraph wGT3x-BT for step counting during inpatient spinal cord rehabilitation
}

\author{
Erin Albaum ${ }^{1,2} \cdot$ Emily Quinn $^{1,2} \cdot$ Saba Sedaghatkish ${ }^{1,2} \cdot$ Parminder Singh $^{1,2} \cdot$ Amber Watkins $^{1,2} \cdot$ \\ Kristin Musselman $\mathbb{1}^{1,2} \cdot$ Josh Williams ${ }^{1,2}$
}

Received: 9 October 2018 / Revised: 2 January 2019 / Accepted: 18 January 2019 / Published online: 8 February 2019

(c) International Spinal Cord Society 2019

\begin{abstract}
Study design Cross-sectional.

Objectives (1) Assess the accuracy of the Actigraph wGT3x-BT accelerometer to count steps taken by inpatients with incomplete spinal cord injury (iSCI) in physical therapy (PT) sessions and self-directed activities, and (2) compare the number of steps/min taken in PT sessions to that in self-directed activities during inpatient rehabilitation.

Setting Inpatient spinal cord injury rehabilitation.

Methods Seventeen individuals with subacute motor iSCI were observed for up to 45-min of both PT and self-directed activities, during which steps were simultaneously tracked by the Actigraph wGT3x-BT and a researcher using a hand tally counter. Accuracy was evaluated with an intraclass correlation coefficient (ICC) for the entire PT session and self-directed activities, as well as for periods of walking.

Results There was excellent agreement between the Actigraph wGT3x-BT and manually counted steps for entire PT sessions (ICC $=0.86$ ) and walking periods ( $\mathrm{PT}$ walking, $\mathrm{ICC}=0.99$; self-directed walking, $\mathrm{ICC}=0.99$ ). There was poor agreement for entire self-directed sessions (ICC $=0.15$ ). Visual analysis of Bland-Altman plots supported these findings. Participants took more steps/min in PT sessions compared to self-directed activities $(p=0.023)$.

Conclusion The Actigraph wGT3x-BT accurately counts steps during PT sessions and walking periods in individuals with subacute motor iSCI. Clinically, this may enable physical therapists to track walking repetitions during inpatient rehabilitation more effortlessly.
\end{abstract}

\section{Introduction}

Individuals with motor incomplete spinal cord injury (iSCI) have the potential to regain functional ambulation through neuroplastic change [1]. It is well documented that recovery of walking ability is most significant in the first year following injury and is influenced by the volume of walking training $[1,2]$. Mass practice of stepping that involves many movement repetitions leads to greater walking gains for

Josh Williams

josh.williams@utoronto.ca

1 Department of Physical Therapy, University of Toronto, Toronto, ON, Canada

2 Toronto Rehabilitation Institute, University Health Network, Toronto, ON, Canada individuals with iSCI than interventions that do not emphasize repetitive stepping [3].

Since regaining the ability to walk is a top priority amongst individuals with iSCI, their families and treating clinicians [4], walking retraining is the focus of inpatient rehabilitation for many individuals with motor iSCI $[5,6]$. However, the amount of walking practice received during physical therapy (PT), whether recorded as time or number of steps, is rarely tracked [5, 6]. Documenting the volume of walking training could enable clinicians and patients to track movement repetitions and monitor progress. Moreover, training volume is a metric that may be used to reflect the quality of rehabilitation after iSCI and to advocate for changes to the health system [2, 7]. In fact, accreditation standards for inpatient SCI rehabilitation centres are currently being developed and implemented in Canada, with the volume of training identified as a key indicator for walking [7]. 
Manually measuring the amount of time patients spend walking or the number of steps they take during therapy are onerous tasks for clinicians. Accelerometers have been used to assess repetitions of movement as a measure of walking volume in a variety of populations with altered gait patterns and speeds, such as geriatrics [8], Parkinson's disease [9], multiple sclerosis [10], stroke [11], and iSCI [12]. More specifically, Zbogar et al. (2016) found moderate to high test-retest reliability of wrist and hip accelerometers over 2 days in individuals with SCI during inpatient rehabilitation [13]. A moderate correlation was also found between the wrist accelerometer and grip strength, and the hip accelerometer and measures of walking [13]. Additionally, Ishikawa et al. (2011) used the Step Activity Monitor to track step counts in 11 individuals with iSCI over 7 consecutive days [14]. This study showed that a minimum of 2 days was required to reliably report average step counts using the Step Activity Monitor in the iSCI population [14]. Although this research gives insight into the clinical use of accelerometers to measure activity levels in individuals with iSCI, neither of these studies compare the step counts obtained by the accelerometers to a gold standard (i.e. manually counted steps) in order to establish criterion validity. Bowden and Behrman (2007) and Treacy et al. (2018) assessed the step count accuracy of the StepWatch Activity Monitor compared to hand-tallied steps during various standardized tests, such as the 6-min walk test $(6 \mathrm{mWT})$ and $10-\mathrm{min}$ walk test $(10 \mathrm{mWT})$ in a variety of populations with slow gait speeds $[12,15]$. Although these studies demonstrated criterion validity of the StepWatch Activity Monitor, these findings may not be generalizable for prolonged periods of time considering that accuracy was only assessed during standardized walking tests. For example, a full PT session is vastly different than a standardized walking test as a variety of movements including walking and non-walking tasks are performed. Comparing accelerometer steps to manual count during full PT sessions may have greater clinical relevance than monitoring steps during standardized tests. Criterion validation of a step count tool during PT sessions in the iSCI population is a critical first step to its use as a measure of volume of walking during inpatient iSCI rehabilitation.

One triaxial accelerometer that has been used to measure physical activity is the Actigraph wGT3x-BT. This monitor is also capable of recording the number of steps taken using an algorithm in the associated software program; however, there has been little investigation into the accuracy of this device and algorithm to quantify the number of steps taken in individuals with damage to the central nervous system. Recently, Webber and St John (2016) examined the accuracy of the Actigraph wGT3x-BT to record step counts in geriatric patients admitted to an inpatient rehabilitation unit [8]. The findings of this study indicated that when placed on the ankle, but not the hip, the Actigraph wGT3x-BT accurately measured step counts in individuals with slow walking speeds [8]. This device is also less expensive than the Step Activity Monitor and may therefore be more clinically feasible. For these reasons, it is worthwhile to investigate the Actigraph wGT3x-BT at the ankle of individuals with iSCI.

Research on the accuracy of these devices during selfdirected activities is also limited. Considering inpatients with SCI spend the majority of their time outside of PT, measuring step counts during this time may give a more holistic representation of volume of walking achieved during inpatient rehabilitation [16]. Past literature has used various accelerometers to investigate self-directed activity levels in both elderly and SCI populations; however current research has not compared device step counts to manual counts to ensure accuracy during self-directed time [16-18]. Currently, there is no device that has been validated as an accurate measure of step counts in both PT and self-directed activities in the iSCI population.

Given the need for a tool to measure the volume of walking practice during inpatient iSCI rehabilitation, the primary aim of this study was to evaluate the accuracy (i.e. criterion validity) of the Actigraph wGT3x-BT to measure step counts in adults with subacute motor iSCI during PT sessions and self-directed activities. We hypothesized that there will be excellent agreement (i.e. intraclass correlation coefficient $[\mathrm{ICC}] \geq 0.80$ ) between the Actigraph wGT3x-BT and manually counted steps during both PT sessions and self-directed activities. Information on the types of activities carried out during PT and self-directed activities were recorded to explore sources of error if the Actigraph wGT3x-BT was found to be inaccurate. If accurate, this device may provide physical therapists with an efficient way of measuring and documenting walking volume in the iSCI population during and outside of PT. In addition, we were interested in gaining a better understanding of the steps/ minute taken outside of PT, as this unstructured time provides an opportunity for patients to maximize the number of daily steps. Thus, the secondary aim of this study was to compare the mean number of steps/minute taken by adults with subacute motor iSCI during PT sessions and selfdirected activities. We hypothesized that individuals with subacute motor iSCI will take a greater number of steps during PT sessions compared to self-directed activities.

\section{Methods}

\section{Participants}

Inpatients of the Toronto Rehabilitation Institute-University Health Network-Lyndhurst Centre (Toronto, 
Ontario, Canada) who met the following criteria were eligible to participate in this study: (1) sustained a motor iSCI within the last 6 months, (2) aged 18 years or older, (3) classified as either American Spinal Injury Association Impairment Scale (AIS) C or D by the International Standards for Neurological Classification of Spinal Cord Injury (ISNCSCI) [19], (4) scored greater than 2C on the Rick Hansen Spinal Cord Injury Registry (RHSCIR) [20] Standing and Walking Assessment Tool (i.e. requires $<25 \%$ physical assistance for ambulation with or without an assistive device and/or orthoses), and (5) did not have any condition other than iSCI that may have impaired walking ability (e.g. vestibular deficit, orthopedic injury, moderate, or severe brain injury). Participants were recruited through convenience sampling; physical therapists treating the target population referred potential participants. The time of data collection was based around participant convenience as opposed to a specific point during inpatient rehabilitation (see Table 1). Participants provided informed written and verbal consent prior to participating in the study. Ethics approval for this study was granted by the Research Ethics Boards of the University Health Network and the University of Toronto.

The sample size for this cross-sectional quantitative study was based on the ICC determined from a previous study, in which the same activity monitor was utilized in a rehabilitation setting for geriatric patients [8]. Assuming an ICC of at least 0.938 and a desired confidence interval of 95\%, a sample size of 13 participants was calculated [21].

\section{Procedures}

Each participant wore the Actigraph wGT3x-BT accelerometer (Actigraph, Pensacola, FL; $4.6 \mathrm{~cm} \times 3.3 \mathrm{~cm} \times$ $1.5 \mathrm{~cm} ; 19 \mathrm{~g}$ ) during PT sessions and self-directed activities. The device was placed proximal to the lateral malleolus on the leg least affected by their iSCI (as determined by selfreport) and secured using Velcro straps. A generic hand tally counter was used to manually count steps.

\section{PT session}

Volume of walking was tracked during one session of individualized therapy with a registered physical therapist. The duration of the PT sessions was based on the 45-min time slot allotted at this facility. The median session time for PT sessions was 33.2 min (interquartile range: 28.6, 37.0). A typical PT session was selected; sessions with limited ambulation training, such as those including extensive assessment, education or discharge planning were not included. The Actigraph wGT3x-BT was donned at the beginning of the therapy session and removed promptly upon its completion. Participants' steps were tracked by the
Table 1 Participant demographics and clinical characteristics

\begin{tabular}{|c|c|c|}
\hline Characteristics & Median (25th, 75th percentile) & \\
\hline Age & $62.0(41.5,78.5)$ & \\
\hline \multirow[t]{2}{*}{ Days post-rehab admission } & $20.0(12.5,41.0)$ & \\
\hline & $n$ & $\%$ \\
\hline \multicolumn{3}{|l|}{ Sex } \\
\hline Male & 13 & 76.5 \\
\hline Female & 4 & 23.5 \\
\hline \multicolumn{3}{|l|}{ AIS classification } \\
\hline $\mathrm{C}$ & 0 & 0 \\
\hline $\mathrm{D}$ & 17 & 100 \\
\hline \multicolumn{3}{|l|}{ RHSCIR stage } \\
\hline $2 \mathrm{C}$ & 1 & 5.9 \\
\hline $3 \mathrm{~A}$ & 1 & 5.9 \\
\hline $3 \mathrm{~B}$ & 1 & 5.9 \\
\hline $3 \mathrm{C}$ & 13 & 76.5 \\
\hline 4 & 1 & 5.9 \\
\hline \multicolumn{3}{|l|}{ Etiology } \\
\hline Traumatic & 5 & 29.4 \\
\hline Non-traumatic & 12 & 70.6 \\
\hline & $n$ & \\
\hline \multicolumn{3}{|l|}{ Gait aid ${ }^{\mathrm{a}}$} \\
\hline Rollator walker & 14 & \\
\hline Two-wheeled walker & 1 & \\
\hline None & 2 & \\
\hline
\end{tabular}

${ }^{\mathrm{a}}$ Gait aid used during self-selected activities

AIS American Spinal Injury Association Impairment Scale; RHSCIR Rick Hansen Spinal Cord Injury Registry. RHSCIR stage is listed from lower walking function (i.e. 2C) to higher walking function (i.e. 4). $2 \mathrm{C}=$ ability to stand and initiate reciprocal steps through voluntary lower extremity movement but requires minimal physical assistance ( $<25 \%$ of total effort) of one person and may include use of assistive devices and/or orthoses with the exception of bilateral knee-anklefoot orthoses (KAFOs); $3 \mathrm{~A}=$ ability to ambulate daily using reciprocal steps over ground for short distances $(10-100 \mathrm{~m})$ with supervision. Person may use assistive devices and/or orthoses with the exception of bilateral KAFOs; $3 \mathrm{~B}=$ ability to ambulate daily using reciprocal steps over ground for short distances $(10-100 \mathrm{~m})$ independently. Person may use assistive devices and/or orthoses with the exception of bilateral KAFOs; $3 \mathrm{C}=$ ability to ambulate daily using reciprocal steps over ground for long distances $(>100 \mathrm{~m}$ ) independently. Person may use assistive devices and/or orthoses with the exception of bilateral KAFOs; $4=$ ability to ambulate full time daily at home and in the community without assistive devices, orthoses, or physical assistance.

Actigraph wGT3x-BT and simultaneously counted manually by a researcher using a hand tally counter during each session. The type and duration of activities performed during each session were also recorded to the second and classified as either a walking or non-walking task. All tasks carried out during the PT sessions and self-directed activities were recorded to the second using the clock from the same computer used to initialize the Actigraph wGT3x-BT. 
For the purpose of manually counting, a step was defined as a movement in which one foot was lifted from the ground and subsequently set down in a new position. Steps were manually counted for activities classified as a walking task during which participants took purposeful steps for locomotion. For the purpose of this study the following were considered non-walking tasks: bed exercises, elliptical, NuStep, seated exercises, sitting, sleeping, standing balance (i.e., therapeutic exercises that challenged balance in various stances), standing exercises (i.e., therapeutic exercises for lower extremity strengthening), static standing (i.e., nontherapeutic standing with or without therapist support and/ or gait aid) and stationary bike. The total number of manually counted steps was recorded upon completion of each observed session.

\section{Self-directed activities}

Participants were observed by a researcher for up to 45-min during self-directed activities to be consistent with the amount of time offered in a PT session. The median session time for self-directed activities was $39.0 \mathrm{~min}$ (Interquartile range: $30.0,42.9)$. The observation session was scheduled based on participant preference and to minimize sedentary activities (i.e. sleeping) in order to avoid any potential analytical bias of the Actigraph wGT3x-BT towards greater agreement. Step counts were simultaneously obtained using the Actigraph wGT3x-BT and hand tally counter in an identical manner to the PT session.

\section{Data analysis}

All data from the Actigraph wGT3x-BT were downloaded using ActiLife software (version 6.13.3; Actigraph, Pensacola, FL) with the low-frequency extension and one-second epoch settings selected to increase sensitivity for slower gait speeds [8]. Actigraph wGT3x-BT step counts were obtained using the step count algorithm in the ActiLife software. Data were then exported into a Microsoft Excel spreadsheet that displayed the number of steps per second of activity.

Actigraph wGT3x-BT counted steps and manually counted steps for both the PT session and the self-directed activities were divided by the total session time and expressed as steps/min. The sessions were subsequently divided into walking and non-walking tasks. For each participant, the steps captured by the Actigraph wGT3x-BT and the steps counted manually during walking tasks were divided by the total time spent walking and reported as the number of steps/min in walking tasks. Additionally, the percentage of time spent walking in PT sessions and in selfdirected activities was reported. For non-walking tasks, the manual count was recorded as zero. Movements picked up by the Actigraph wGT3x-BT during non-walking tasks were divided by the total time spent in that task and reported as steps/min taken during each of the non-walking tasks. All step data were reported as steps/min to normalize for time differences in both PT sessions and self-directed activities among the participants.

\section{Statistical analyses}

Statistical analyses were conducted using SPSS Statistics, Version 22 (IBM Corp., Armonk, NY). The Shapiro Wilks test was used to assess normality and an alpha value of 0.05 was used for all tests. All normally distributed data are presented with a mean and standard deviation, while all data not normally distributed are presented with a median and 25 th and 75 th percentiles. ICCs (two-way random model, absolute agreement), reported with the $95 \%$ confidence interval, were used to assess agreement between the Actigraph wGT3x-BT and manually counted steps for the full PT sessions and self-directed activities, as well as for walking tasks during each session. Excellent agreement was defined as an ICC of $\geq 0.80$, good agreement as $0.70-0.79$ and poor agreement as $<0.7$ [22]. Bland-Altman plots were generated to display the agreement between the Actigraph wGT3x-BT counted steps and manually counted steps. The difference between the two measures was plotted on the $y$ axis, and the mean number of steps/min ([Actigraph wGT3x-BT + manually counted steps]/2) on the $x$-axis. If the difference was normally distributed, the mean difference and $95 \%$ limits of agreement were plotted. If the difference was not normally distributed, the median difference and 2.5th and 97.5th percentiles were plotted [23].

Manually counted steps, expressed as steps/min, were compared between PT and self-directed activities with a paired $t$-test for normally distributed data or a Wilcoxon rank sum test for non-normally distributed data. Similarly, the percentage of time spent walking during PT sessions versus self-directed activities was compared using a paired sample $t$-test or a Wilcoxon rank sum test as appropriate. The Bonferroni correction for multiple comparisons was applied, resulting in an alpha value of 0.025 .

\section{Results}

\section{Participants}

A total of 19 inpatients with iSCI met the eligibility criteria and provided informed consent. Data were collected and available for 17 participants for PT sessions and 13 participants for self-directed activities. (see Fig. 1). The 13 individuals included in the analysis of self-directed activities were the same participants included in the comparison of median steps/min in PT and self-directed activities 


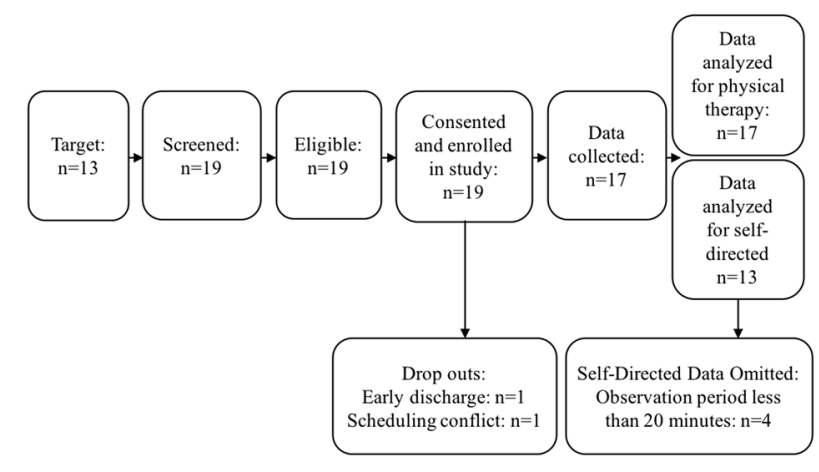

Fig. 1 Participant recruitment chart

(secondary objective). Participant demographics are presented in Table 1.

\section{Accuracy of Actigraph wGT3x-BT}

Excellent agreement was observed between the Actigraph wGT3x-BT and the manually counted steps for the entire PT session (ICC $=0.86,95 \%$ CI $[0.49,0.95]$ ), periods of walking during PT (ICC $=0.99,95 \%$ CI $[0.96,1.00])$, and selfdirected activities (ICC $=0.99,95 \%$ CI $[0.95,1.00]$ ). The observed agreement during the entire period of self-directed activities was poor (ICC $=0.15,95 \%$ CI $[-0.18,0.55])$.

Visual analysis of the Bland-Altman plots supports the resulting ICC values. A positive bias is observed for the entire periods of PT and self-directed activities indicating the Actigraph wGT3x-BT tended to over-count steps; the mean difference between Actigraph wGT3x-BT and manually counted steps/min is above zero with wide limits of agreement (Fig. 2a, c). In comparison, the Bland-Altman plots for walking tasks during PT and self-directed activities showed a mean difference between measures near zero with narrow limits of agreement (Fig. 2b, d).

\section{Steps during PT and self-directed activities}

A greater percentage of time was spent walking during PT $(48.6 \pm 14.9 \%)$ than during self-directed sessions $(24.7 \pm$ $13.7 \%), t(12)=4.42, p=0.001$. Additionally, there was a significant difference between the median number of manually counted steps/min during PT (23.2 steps/min $[14.9,31.6 \mathrm{steps} / \mathrm{min}])$ compared to self-directed sessions (13.3 steps/min [8.9, 22.7 steps $/ \mathrm{min}]), Z=-2.27, p=$ 0.023 , with more steps/min taken during PT.

\section{Non-walking tasks during PT and self-directed activities}

Non-walking tasks completed during PT sessions and selfdirected activities are listed in Table 2. During PT, mean percent time amongst all participants was greatest for sitting
$(19.2 \pm 16.9 \%)$ followed by bed exercises $(15.2 \pm 12.9 \%)$. In comparison, during self-directed activities, mean percent time amongst all participants was greatest for sitting (25.4 \pm $25.4 \%$ ), followed by the NuStep, a recumbent cross trainer $(14.6 \pm 28.0 \%)$. Regardless of setting, stationary cardiovascular exercises, including the NuStep, elliptical and stationary bike registered the highest number of median steps/min by the Actigraph wGT3x-BT (Table 2).

\section{Discussion}

This study evaluated the accuracy (i.e. criterion validity) of the Actigraph wGT3x-BT to count steps during PT sessions and self-directed activities in individuals with subacute motor iSCI. Consistent with our primary hypothesis, the Actigraph wGT3x-BT was found to have excellent agreement with manually counted steps during PT sessions, as well as during walking tasks in both PT and self-directed activities. However, poor agreement was observed between the Actigraph wGT3x-BT and manually counted steps when considering the entirety of self-directed activities. Participants took a greater number of steps/min during PT sessions compared to self-directed activities, supporting our secondary hypothesis. These findings indicate the types of activities and settings in which the Actigraph wGT3x-BT can be used to accurately report step counts.

Although there was less time spent walking during selfdirected activities compared to PT sessions, the breakdown of tasks shows that participants did in fact engage in lower extremity movement repetitions through stationary cardiovascular exercises, such as the stationary bike. However, on average participants spent the greatest proportion of time sitting during self-directed activities. This finding is similar to that of Zbogar et al., who found that inpatients with SCI spend between $45 \%$ and $50 \%$ of their time engaged in sedentary activities [16]. Considering that the majority of patient time is spent outside of PT, there is considerable opportunity to further increase the number of steps and lower extremity movement repetitions in individuals with iSCI during self-directed time to maximize neuroplastic change $[16,24]$.

Engagement in non-stepping lower extremity repetitions, such as cycling on a stationary bike, resulted in steps being incorrectly counted by the Actigraph wGT3x-BT. Placement of the accelerometer at the hip rather than the ankle may have eliminated this inaccuracy. However, previous work in older adults with slow gait speeds demonstrated that placement of the Actigraph wGT3x-BT at the hip is less accurate at counting steps than placement at the ankle [8]. More specifically, Webber and St. John found that placement at the hip resulted in a large number of steps being missed during ambulation [8]. 


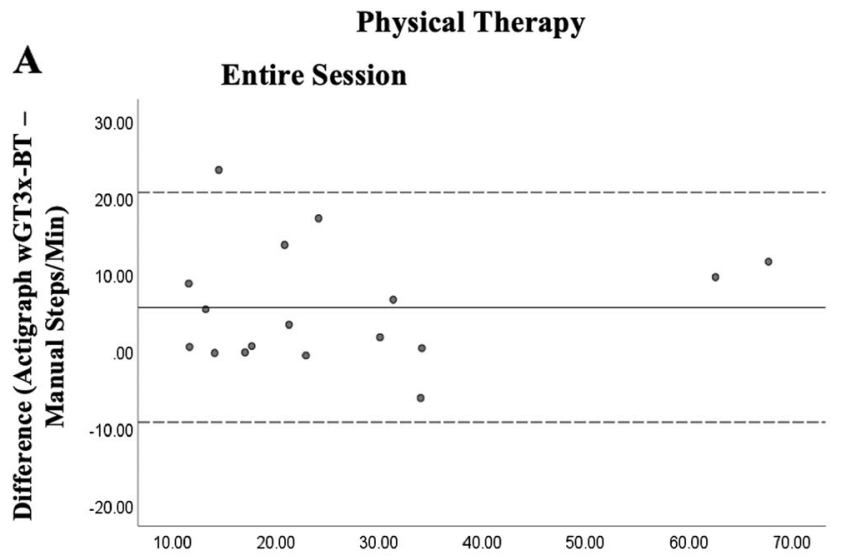

Mean Steps/Min [(Actigraph wGT3x-BT + Manual)/2]

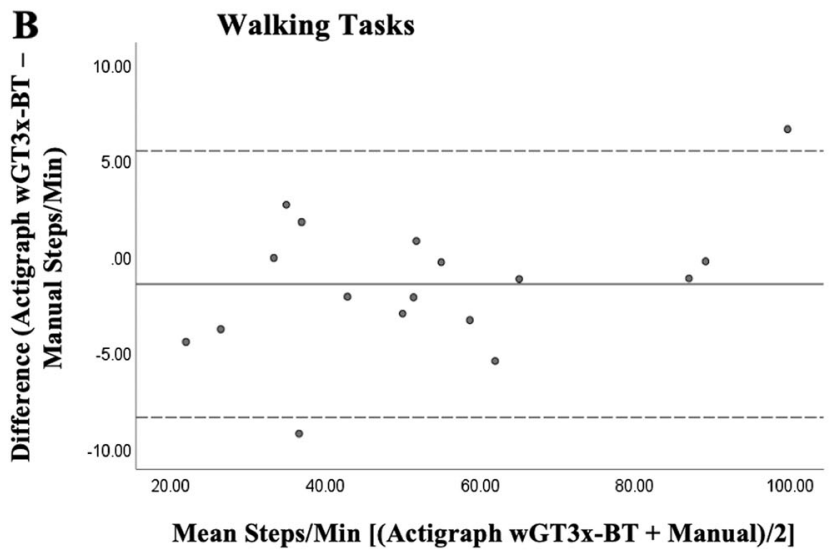

Fig. 2 Bland-Altman plots of the difference between Actigraph wGT3x-BT and manually counted steps/min for (a) entire physical therapy sessions, (b) walking tasks during physical therapy sessions,
Self-Directed
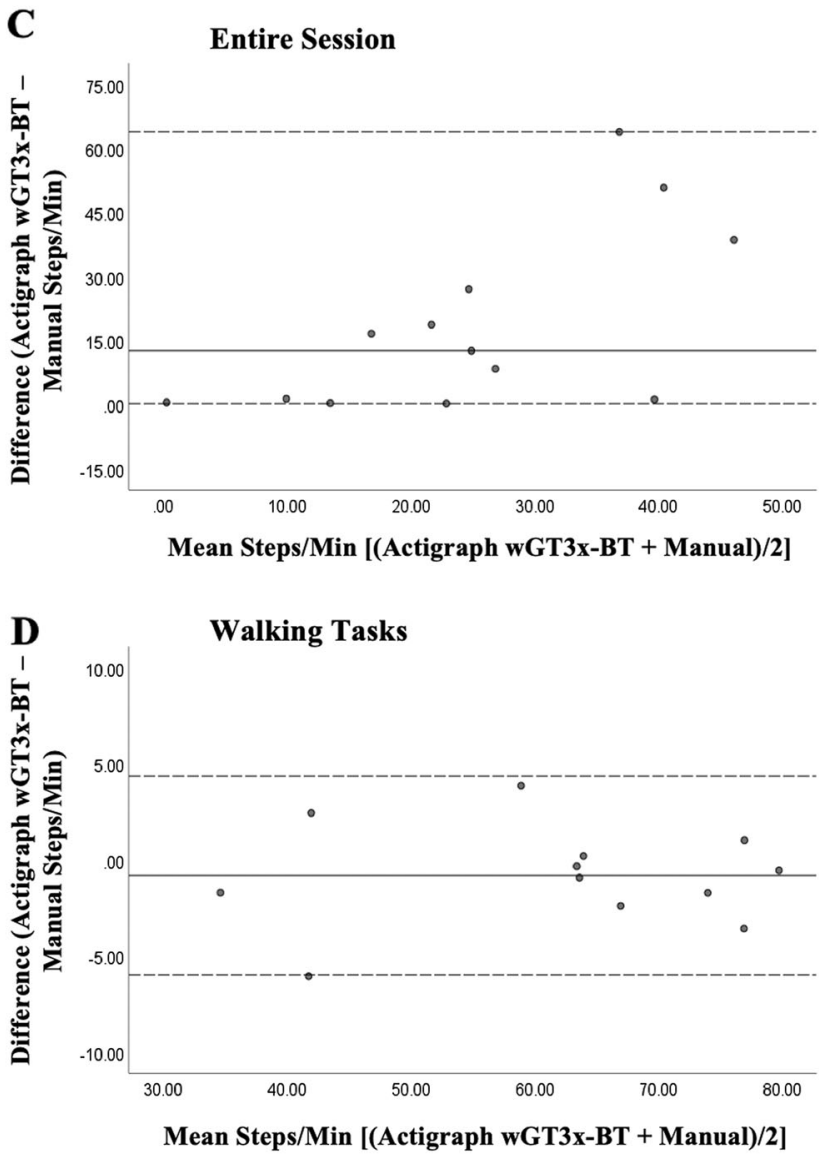

(c) entire self-directed session, and (d) walking tasks during selfdirected sessions. Solid line $=$ mean difference; dashed lines $=$ upper and lower limits of agreement for $95 \%$ confidence interval
Table 2 Actigraph GT3x-BT summary statistics for tasks completed during physical therapy sessions and selfdirected activities

\begin{tabular}{|c|c|c|c|c|c|c|}
\hline \multirow[t]{2}{*}{ Task } & \multirow[t]{2}{*}{$n$} & \multicolumn{2}{|c|}{ Physical therapy } & \multirow[t]{2}{*}{$n$} & \multicolumn{2}{|l|}{ Self-directed } \\
\hline & & $\begin{array}{l}\text { Mean \% time } \\
( \pm \mathrm{SD})\end{array}$ & $\begin{array}{l}\text { Median steps/min } \\
\text { (25th, 75th } \\
\text { percentile) }\end{array}$ & & $\begin{array}{l}\text { Mean \% time } \\
( \pm \text { SD })\end{array}$ & $\begin{array}{l}\text { Median steps/min } \\
\text { (25th, 75th } \\
\text { percentile) }\end{array}$ \\
\hline Walking & 17 & $48.6 \pm 14.9$ & $23.2(14.9,31.6)$ & 13 & $24.7 \pm 13.7$ & $13.3(8.9,22.7)$ \\
\hline Bed exercises & 11 & $15.2 \pm 12.9$ & $6.5(4.7,17.1)$ & 2 & $8.1 \pm 8.1$ & $1.5(1.1,-)$ \\
\hline Elliptical & 1 & $0.8 \pm 2.9$ & $52.1(52.1,52.1)$ & 0 & 0 & N/A \\
\hline NuStep & 2 & $2.8 \pm 9.5$ & $47.3(42.8,-)$ & 4 & $14.6 \pm 28.0$ & $66.6(52.4,75.7)$ \\
\hline $\begin{array}{l}\text { Seated } \\
\text { exercises }\end{array}$ & 2 & $2.7 \pm 9.5$ & $8.6(3.5,-)$ & 3 & $4.8 \pm 10.2$ & $20.0(18.3,-)$ \\
\hline Sitting & 16 & $19.2 \pm 16.9$ & $4.9(3.4,12.1)$ & 7 & $25.4 \pm 25.4$ & $4.5(1.9,16.2)$ \\
\hline Sleeping & 0 & 0 & N/A & 2 & $13.5 \pm 32.0$ & $3.4(2.5,-)$ \\
\hline $\begin{array}{l}\text { Standing } \\
\text { balance }\end{array}$ & 3 & $2.4 \pm 6.8$ & $4.5(2.5,-)$ & 0 & 0 & N/A \\
\hline $\begin{array}{l}\text { Standing } \\
\text { exercises }\end{array}$ & 6 & $2.4 \pm 4.8$ & $4.9(1.3,15.5)$ & 0 & 0 & N/A \\
\hline Static standing & 3 & $5.0 \pm 5.3$ & $0(0,-)$ & 1 & $0.1 \pm 0.38$ & $27.3(27.3,27.3)$ \\
\hline Stationary bike & 1 & $0.8 \pm 2.9$ & $32.3(32.3,32.3)$ & 3 & $8.9 \pm 17.8$ & $55.5(40.5,-)$ \\
\hline
\end{tabular}


The number of steps/min observed during PT sessions in this study (23.2 steps/min [14.9, 31.6 steps/min]) equates to more than 500 steps taken in a PT session. This number of steps is considerably higher than the values reported by Zbogar et al. in a previous study involving two Canadian rehabilitation facilities [6]. They reported fewer steps being taken by ambulatory inpatients with SCI during PT and occupational therapy sessions: a median of 51 steps/session (interquartile range: 0-176 steps) at admission to rehabilitation and a median of 115 steps (interquartile range: $21-313$ steps) at discharge. The discrepancy in the findings of the two studies may be due to differences in the study samples. While participants in both studies included primarily individuals with AIS D SCI, the participants in the study by Zbogar and colleagues had lower walking function, as demonstrated by the low mean score on the Walking Index for Spinal Cord Injury II (WISCI II) (i.e. 6.4 \pm 7.9 ). The majority of participants in the current study would have scored 13 on the WISCI II (ambulates with a walking, no braces and no physical assistance) [25], with a few scoring 9 due to the use of an ankle-foot orthosis in addition to a walker. However, the findings from both studies suggest that the number of steps taken during inpatient PT sessions in Canada falls short of the number of repetitions observed during mass practice of stepping [3].

\section{Study limitations}

Firstly, the study sample is only partly representative of the Canadian SCI population. The majority of our participants were male, which aligns with the Canadian estimate that $79.1 \%$ of individuals living with SCI are male [26]; however, the majority of our participants experienced a nontraumatic SCI. Recent estimates suggest that non-traumatic injuries represent approximately half of all SCI in Canada [27]. Considering that non-traumatic SCI most commonly occurs in individuals between 55 and 85 years of age and the median age of the study participants was 62 years, it is not surprising that the majority of SCI diagnoses were nontraumatic [27]. Secondly, participants had similar diagnoses and walking abilities (i.e. 100\% AIS D and $76.5 \%$ staged 3C according to the RHSCIR Standing and Walking Assessment Tool, respectively), which increases the internal validity of the findings, but results in low external validity, thereby limiting the generalizability of the findings to the larger iSCI population. Thirdly, the presence of an observer may have motivated both the physical therapists and participants to perform more walking tasks during PT and selfdirected activities compared to unobserved periods. Finally, scheduling of self-directed time was patient-driven, and observation limited to 45-min due to scheduling constraints.
Consequently, our findings may not accurately reflect the types of activities that individuals perform over the course of an entire day.

\section{Future directions}

Future research should investigate if the Actigraph wGT3xBT accurately records the number of revolutions/min for activities, such as the NuStep or stationary bike. If accurate, the Actigraph wGT3x-BT may instead be used to monitor lower extremity movement repetitions in addition to step counts to provide a measure of training volume in the iSCI population.

\section{Conclusions}

This study provides insight into the clinical application of the Actigraph wGT3x-BT in the iSCI population during PT sessions and self-directed activities. Specifically, it is an accurate device to count steps during walking tasks, regardless of setting.

\section{Data archiving}

The datasets generated and/or analyzed during the current study are available from the corresponding author on reasonable request.

Acknowledgements The authors would like to thank the participants that took part in this study, the physiotherapy staff at Toronto Rehabilitation Institute-University Health Network-Lyndhurst Centre and specifically Sarah Fingland PT for her help with participant recruitment.

Funding This study was supported by the Toronto Rehabilitation Institute-University Health Network.

Author contribution EA was responsible for conducting a literature review, designing and writing the research protocol, data collection and analysis, interpretation of results, writing the manuscript, generating tables and figures and updating reference lists. EQ was responsible for conducting a literature review, designing and writing the research protocol, screening eligible participants, data collection and writing the manuscript. SS was responsible for screening eligible participants, data collection and analysis and generating tables and figures. PS was responsible for conducting a literature review, designing and writing the research protocol, data collection and analysis, interpretation of results and statistical analysis. AW was responsible for conducting a literature review, designing and writing the research protocol, screening eligible participants, data collection, and writing the manuscript. $\mathrm{KM}$ was responsible for designing and writing the research protocol, interpretation of results, statistical analysis and writing the manuscript. JW was responsible for designing and writing the research protocol, interpretation of results and writing the manuscript. 


\section{Compliance with ethical standards}

Conflict of interest The authors declare that they have no conflict of interest.

Ethical approval This study was performed in accordance with the Declaration of Helsinki and was approved by the Research Ethics Boards of the University Healthy Network (\#17-5980) and the University of Toronto. We certify that all applicable institutional and governmental regulations concerning the ethical use of human volunteers were followed during the course of this research.

Publisher's note: Springer Nature remains neutral with regard to jurisdictional claims in published maps and institutional affiliations.

\section{References}

1. Burns AS, Marino RJ, Flanders AE, Flett H. Clinical diagnosis and prognosis following spinal cord injury. Handb Clin Neurol. 2012;109:47-62.

2. Yang JF, Musselman KE. Training to achieve over ground walking after spinal cord injury: a review of who, what, when, and how. J Spinal Cord Med. 2012;35:293-304

3. Yang JF, Musselman KE, Livingstone D, Brunton K, Hendricks G, Hill D, et al. Repetitive mass practice or focused precise practice for retraining walking after incomplete spinal cord injury? A pilot randomized clinical trial. Neurorehabil Neural Repair. 2014;28:314-24.

4. Anderson KD. Targeting recovery: priorities of the spinal cordinjured population. J Neurotrauma. 2004;21:1371-83.

5. Taylor-Schroeder S, LaBarbera J, McDowell S, Zanca JM, Natale A, Mumma S, et al. Physical therapy treatment time during inpatient spinal cord injury rehabilitation. J Spinal Cord Med. 2011;34:149-61.

6. Zbogar D, Eng JJ, Miller WC, Krassioukov AV, Verrier MC. Movement repetitions in physical and occupational therapy during spinal cord injury rehabilitation. Spinal Cord. 2017;55:172-9.

7. Craven C, Flett H, Hitzig S, Farahani F, Alavinia M, Omidvar M, et al. Moving from the prioritization to implementation: the spinal cord injury rehabilitation care high performance indicators (scihigh) project. J Spinal Cord Med. 2017;40:813-69.

8. Webber SC, St John PD. Comparison of ActiGraph GT3X + and StepWatch step count accuracy in geriatric rehabilitation patients. J Aging Phys Act. 2016;24:451-8.

9. Dijkstra B, Zijlstra W, Scherder E, Kamsma Y. Detection of walking periods and number of steps in older adults and patients with Parkinson's disease: accuracy of a pedometer and an accelerometry-based method. Age Ageing. 2008;37:436-41.

10. Sandroff BM, Motl RW, Pilutti LA, Learmonth YC, Ensari I, Dlugonski D, et al. Accuracy of StepWatch ${ }^{\mathrm{TM}}$ and ActiGraph accelerometers for measuring steps taken among persons with multiple sclerosis. PLoS One. 2014;9:e93511.
11. Campos C, DePaul VG, Knorr S, Wong JS, Mansfield A, Patterson KK. Validity of the ActiGraph activity monitor for individuals who walk slowly post-stroke. Top Stroke Rehabil. 2018;25:295-304.

12. Bowden MG, Behrman AL. Step activity monitor: accuracy and test-retest reliability in persons with incomplete spinal cord injury. J Rehabil Res Dev. 2007;44:355-62.

13. Zbogar D, Eng JJ, Miller WC, Krassioukov AV, Verrier MC. Reliability and validity of daily physical activity measures during inpatient spinal cord injury rehabilitation. SAGE Open Med. 2016;4:1-9.

14. Ishikawa S, Stevens SL, Kang M, Morgan DW. Reliability of daily step activity monitoring in adults with incomplete spinal cord injury. J Rehabil Res Dev. 2011;48:1187-94.

15. Treacy D, Hassett L, Schurr K, Chagpar S, Paul SS, Sherrington C. Validity of different activity monitors to count steps in an inpatient rehabilitation setting. Phys Ther. 2017;97:581-8.

16. Zbogar D, Eng JJ, Miller WC, Krassioukov AV, Verrier MC. Physical activity outside of structured therapy during inpatient spinal cord injury rehabilitation. J Neuroeng Rehabil. 2016;13:99.

17. Arnardottir NY, Koster A, Van Domelen DR, Brychta RJ, Caserotti P, Eiriksdottir G, et al. Objective measurements of daily physical activity patterns and sedentary behaviour in older adults: age, gene/environment susceptibility-reykjavik study. Age Ageing. 2013;42:222-9.

18. Nurdiana A, Brown WJ, Clark B, Ahmad Munir CM, Singh Rabindarjeet. Assessing physical activity levels of elderly malays living in semi-rural areas using tri-axial accelerometer. Malays $\mathbf{J}$ Nutr. 2016;22:363-74.

19. Kirshblum SC, Waring W, Biering-Sorensen F, Burns SP, Johansen M, Schmidt-Read M, et al. Reference for the 2011 revision of the international standards for neurological classification of spinal cord injury. J Spinal Cord Med. 2011;34:547-54.

20. Verrier M, Gagnon D, Musselman K. SCI standing and walking assessment. Rick Hansen Institute. 2017.

21. Streiner DL, Norman GR. Health measurement scales. 4th edn. Oxford: Oxford University Press; 2008.

22. Portney LG, Watkins MP. Foundations of clinical research: applications to practice. 2nd edn. Upper Saddle River: Prentice Hall Health; 2000.

23. Bland JM, Altman DG. Measuring agreement in method comparison studies. Stat Methods Med Res. 1999;8:135-60.

24. Onifer S, Smith G, Fouad K. Plasticity after spinal cord injury: relevance to recovery and approaches to facilitate it. Neurotherapeutics. 2011;8:283-93.

25. Dittuno PL, Dittuno JF. Walking Index for Spinal Cord Injury (WISCI II): scale revision. Spinal Cord. 2001;39:654-6.

26. Wyndaele M, Wyndaele J. Incidence, prevalence and epidemiology of spinal cord injury: what learns a worldwide literature survey? Spinal Cord. 2006;44:523-9.

27. Noonan VK, Fingas M, Farry A, Baxter D, Singh A, Fehlings $\mathrm{MG}$, et al. Incidence and prevalence of spinal cord injury in Canada: a national perspective. Neuroepidemiology. 2012; 38:219-26. 